\title{
Mordeduras de serpientes en un área de explotación minera. Cesar, Colombia. 2017-2019
}

\section{Snake Bites in a Mining Area, Cesar, Colombia. 2017-2019}

Recibido: 04 de septiembre de 2020. Recibido en revisión: 05 de octubre de 2020. Aceptado:13 de noviembre de 2020. DOl : https://doi.org/10.33132/27114260.1906 Muegues Angel Alexis², Redondo Guerra Jessany²

\section{Resumen}

El área de explotación carbonífera en el departamento del Cesar se extiende por cinco municipios, genera cerca de 5100 empleos y vincula 4000 contratistas. La colonización de áreas boscosas con fines de explotación incrementa los casos de mordeduras por serpientes venenosas; el objetivo de esta investigación fue analizar las características epidemiológicas de los accidentes ofídicos reportados al Instituto Nacional de Salud entre 2017 y 2019, provenientes del área de explotación. Resultados: em 54\% de los casos la serpiente murió o fue capturada; las mordeduras se localizaron principalmente en miembros inferiores (74\%) y el tratamiento inmediato consistió en la aplicación de un torniquete compresivo (36\%). La mayoría de los accidentes ocurrieron en Agustín Codazzi (36\%) por las serpientes Bothrops asper (P: 0.00006), Porthidium nasutum (P: 0.00001) y Crotalus durissus (P: 0.0005). Conclusiones: la mordedura de serpiente es un problema de salud pública y requiere la instauración de protocolos de manejo inmediato incluyendo primeros auxilios; las serpientes necesitan ser protegidas por su beneficio a los ecosistemas y el equilibrio medio ambiental.

Palabras claves: Colombia; ecosistemas; minería; serpientes; toxinas.

2 Facultad de Medicina, Fundación Universitaria del Área Andina, Valledupar, Colombia. amuegues(dareandina.edu.co / https:// orcid.org/0000-0002-0836-9262/CvLAC: 0001688944, jessanyr@agmail.com / https://orcid.org/0000-0002-7941-3873 /CvLAC: 0001766449 


\section{Abstract}

The coal mining area in the Cesar department spans five municipalities, creates about 5,100 jobs, and engages 4,000 contractors. The colonization of forested areas for exploitation favors an increase in bites by poisonous snakes; this research intends to analyze the epidemiological characteristics of the ophidian accidents reported to the National Institute of Health between 2017 and 2019 by the mining area. Results. In $54 \%$ of the cases, the snake died or was captured; the bites were mainly located on the lower limbs (74\%), and immediate treatment consisted of applying tourniquet compression (36\%). Most accidents occurred in Agustín Codazzi (36\%), involving the snakes Bothrops asper $(P=0.00006)$, Porthidium nasutum $(P=0.00001)$, and Crotalus durissus $(P=0.0005)$. Conclusions. Snake bites are a public health problem and require immediate mnagement protocols, including first aid. Besides, snakes need to be protected, as they benefit ecosystems and environmental balance.

Keywords: Colombia; ecosystems; mining; snakes; toxins.

\section{Introducción}

Las serpientes son animales ectotérmicos, cuerpo alargado y no tienen extremidades; en Colombia, se distinguen alrededor de 300 especies, lo cual coloca al país entre los diez primeros con mayor cantidad de ofidios (1). Su popularidad se debe a que muchas tienen potencial tóxico, al elaborar cantidades considerables de toxinas, una mezcla de enzimas y proteínas que son peligrosas para los mamíferos $(1,2)$. Estas toxinas son inoculadas a través de un complejo aparato formado por glándulas productoras y colmillos huecos, desarrollados evolutivamente para cazar a sus presas (pequeños roedores, reptiles, aves etc.) pero que también representan peligro para los seres humanos que entran en su hábitat y destruyen sus ecosistemas (2). Cesar es un departamento ubicado en el norte del país con una topografía variada conformada por extensas planicies, zonas boscosas y elevaciones montañosas, siendo todas estas áreas adecuadas para la reproducción de las serpientes. Esta investigación centró su interés en un área de explotación minera de carbón que incluye cinco municipios a saber (Agustín Codazzi, Becerril, Chiruguaná, El Paso y La jagua de Ibirico), debido a que constituye una de las mayores áreas mineras del país, alberga más de cinco 
mil trabajadores formalizados y su área de influencia comprende vastas extensiones de tierra donde son frecuentes los avistamientos de estos animales (3). Los objetivos de este estudio consistieron en calcular la frecuencia de los eventos, las características sociodemográficas de las víctimas, la georreferenciación de los animales y sumar información vital para la conservación de las diferentes especies de ofidios como eslabón fundamental de la cadena alimenticia y el equilibrio medio ambiental.

\section{Metodología}

La aplicación de este diseño de investigación requirió la consulta de la base de datos de los accidentes ofídicos del departamento del Cesar, período 2017-2019, ubicada en la Secretaría de Salud Departamental. Esta fuente de información secundaria recabó datos con ayuda de la ficha para notificación obligatoria número 100 del Ministerio de Salud y la Protección Social. La ficha es diligenciada por médicos tratantes en instituciones de salud de los municipios que conforman el corredor minero y reportada semanalmente al Sistema de Vigilancia Epidemiológica - SIVIGILA. La población estuvo constituida por 278 casos que fueron analizados mediante el programa estadístico Epi-Infor 7, con parámetro de significancia estadística $\mathrm{P}<$
0,05. Epi-Info 7, con el parámetro de significancia estadística $\mathrm{P}<0.05$ (20).

La planeación y ejecución de esta investigación, así como el análisis de la información requirieron una meticulosa revisión ética; acudiendo a las máximas: Ley 911 de 2004 (4), la Resolución 008430, la Resolución 002378 del Ministerio de Salud y la Protección Social (5) y el protocolo de Helsinki (6). Se estableció como de bajo riesgo por no incurrir en lesión física, ni atentados contra la moral o el buen nombre de los individuos víctimas de accidentes ofídicos.

\section{Resultados}

Se mostrarán los resultados partiendo de las variables sociodemográficas; luego, las serpientes más comunes — su georreferenciación-, la atención premédica recibida y finalmente gravedad de los accidentes. El total de pacientes mordidos en el período de estudio fue 278, distribuidos así: 95 en 2017, 88 en 2018 y 95 en 2019. La tasa de incidencia por 100.000 habitantes fue de 4.7 en 2017, 4.5 en 2018 y 4.6 en 2019. El perfil de las víctimas incluye, principalmente, hombres con rango de edad entre 18 y 50 años, de pertenencia étnica mestiza. Una discriminación de las variables sociodemográficas contempladas se resume en la tabla 1. 
Tabla 1. Variables sociodemográficas.

\begin{tabular}{|c|c|c|c|c|}
\hline Variable & Categoría & 2017 & 2018 & 2019 \\
\hline \multirow{4}{*}{ Edad } & $<18$ años & $36 \%$ & $32 \%$ & $29 \%$ \\
\hline & 19-50 años & $48 \%$ & $54 \%$ & $49 \%$ \\
\hline & $51-65$ años & $13 \%$ & $8 \%$ & $15 \%$ \\
\hline & $>66$ años & $3 \%$ & $6 \%$ & $7 \%$ \\
\hline \multirow{3}{*}{ Género } & Masculino & $70 \%$ & $80 \%$ & $76 \%$ \\
\hline & Femenino & $30 \%$ & $20 \%$ & $24 \%$ \\
\hline & Indígena & $14 \%$ & $7 \%$ & $11 \%$ \\
\hline \multirow{3}{*}{ Etnia } & Afrocolombiano & $18 \%$ & $15 \%$ & $27 \%$ \\
\hline & Gitano & $1 \%$ & 0 & 0 \\
\hline & Mestizo & $67 \%$ & $78 \%$ & $62 \%$ \\
\hline \multirow{3}{*}{ Área de ocurrencia } & Cabecera municipal & $34 \%$ & $40 \%$ & $28 \%$ \\
\hline & Rural disperso & $44 \%$ & $41 \%$ & $48 \%$ \\
\hline & Centro poblado & $22 \%$ & $19 \%$ & $24 \%$ \\
\hline
\end{tabular}

Fuente: elaboración propia.

Las serpientes causantes de las ción. Los nombres comunes de estas mordeduras corresponden al género serpientes se encuentran en la tabla 2. Bothrops spp (72\%), seguidas de Cro- Adicionalmente, se encontró que las talus spp (3\%) y Micrurus spp (1\%); $\quad$ serpientes fueron capturadas o sacrifiel $24 \%$ restante no hizo avistamiento cadas en $54 \%$ de los casos. del animal dificultando su identifica-

Tabla 2. Nombres comunes de las serpientes

\begin{tabular}{llccc}
\hline Nombre & Taxonomía & Eventos & $\%$ & Toxina \\
\hline Bocadorada & Bothrops asper & 78 & 28 & \\
Mapaná & Bothrops atrox & 85 & 30 & Hemotóxico y citotóxico \\
Patoco/Patoquilla & Porthidium nasutum & 33 & 12 & \\
Venticuatro & Bothrops atrox & 3 & 1 & Neurotóxico, hemotóxico y \\
Cascabel & Crotalus durissus & 9 & 3 & miotóxico \\
Rabo de ají & Micrurus spp & 3 & 1 & Neurotóxico \\
Sin identificar & --- & 67 & 24 & \\
\hline
\end{tabular}

Fuente: elaboración propia. 
El municipio más afectado es Agustín Codazzi con 99 casos (36\%), seguido de La Jagua de Ibirico con 74 casos (27\%). La tabla 3 muestra el número de eventos en cada uno de los municipios clasificándolos por año.

El cruce de variables "tipo de serpiente" con "municipio de procedencia" demostró algunas diferencias estadísticas significativas: en Agustín Codazzi predominan las mordeduras por "Bocadorada" (P: 0.00006; OR: 2.8, IC: 1.7 4.8), "Patoco" (P: 0.00001; OR: 4.5, IC:
Tabla 3. Accidentes ofídicos por municipio.

\begin{tabular}{lllll}
\hline Municipio & 2017 & 2018 & 2019 & TOTAL \\
\hline Agustín Codazzi & 49 & 31 & 19 & 99 \\
Becerril & 7 & 14 & 15 & 36 \\
Chiruguaná & 4 & 15 & 13 & 32 \\
El Paso & 16 & 9 & 12 & 37 \\
La Jagua de Ibirico & 19 & 19 & 36 & 74 \\
TOTAL & 95 & 88 & 95 & 278 \\
\hline
\end{tabular}

Fuente: elaboración propia.

2.2 - 9.3) y "Cascabel" (P: 0.0005; OR: 7.3, IC: 1.9 - 27). El resumen completo de los resultados se ubica en la tabla 4 .

Tabla 4. Análisis de las mordeduras según el lugar de procedencia

\begin{tabular}{|c|c|c|c|c|c|c|}
\hline \multirow{2}{*}{ Municipio } & & \multicolumn{5}{|c|}{ Bocadorada (Bothrops asper) } \\
\hline & & $\mathrm{Si}$ & No & $\mathbf{P}$ & $X^{2}$ & OR (IC 95\%) \\
\hline \multirow{2}{*}{ Agustín Codazzi } & $\mathrm{Si}$ & 47 & 52 & \multirow{2}{*}{0.00006} & \multirow{2}{*}{14.96} & \multirow{2}{*}{$2.8(1.7-4.8)$} \\
\hline & No & 43 & 136 & & & \\
\hline \multirow{2}{*}{ Becerril } & $\mathrm{Si}$ & 3 & 33 & \multirow{2}{*}{0.0009} & \multirow{2}{*}{9.69} & \multirow{2}{*}{$0.16(0.04-0.5)$} \\
\hline & No & 87 & 155 & & & \\
\hline \multirow{2}{*}{ Chiriguaná } & $\mathrm{Si}$ & 5 & 27 & \multirow{2}{*}{0.031} & \multirow{2}{*}{3.80} & \multirow{2}{*}{$0.35(0.1-0.4)$} \\
\hline & No & 85 & 161 & & & \\
\hline \multirow{2}{*}{ El Paso } & $\mathrm{Si}$ & 12 & 25 & \multirow{2}{*}{0.86} & \multirow{2}{*}{0.02} & \multirow{2}{*}{$1.06(0.5-2.2)$} \\
\hline & No & 78 & 173 & & & \\
\hline \multirow{4}{*}{ La jagua de Ibirico } & $\mathrm{Si}$ & 23 & 51 & \multirow{2}{*}{0.78} & \multirow{2}{*}{0.07} & \multirow{2}{*}{$0.92(0.5-1.6)$} \\
\hline & No & 67 & 137 & & & \\
\hline & \multicolumn{6}{|c|}{ Mapaná (Bothrops atrox) } \\
\hline & & Si & No & $\mathbf{P}$ & $X^{2}$ & OR (IC 95\%) \\
\hline \multirow{2}{*}{ Agustín Codazzi } & $\mathrm{Si}$ & 16 & 83 & \multirow{2}{*}{0.001} & \multirow{2}{*}{10.77} & \multirow{2}{*}{$0.36(0.19-0.67)$} \\
\hline & No & 62 & 117 & & & \\
\hline \multirow{2}{*}{ Becerril } & $\mathrm{Si}$ & 22 & 14 & \multirow{2}{*}{0.000002} & \multirow{2}{*}{22.38} & \multirow{2}{*}{$5.2(2.5-11)$} \\
\hline & No & 56 & 186 & & & \\
\hline
\end{tabular}

(Continúa) 


\begin{tabular}{|c|c|c|c|c|c|c|}
\hline & \multicolumn{6}{|c|}{ Mapaná (Bothrops atrox) } \\
\hline & & Si & No & $\mathbf{P}$ & $x^{2}$ & OR (IC 95\%) \\
\hline \multirow{2}{*}{ Chiriguaná } & $\mathrm{Si}$ & 10 & 22 & \multirow{2}{*}{0.67} & \multirow{2}{*}{0.18} & \multirow{2}{*}{$1.18(0.5-2.6)$} \\
\hline & No & 68 & 178 & & & \\
\hline \multirow{2}{*}{ El Paso } & $\mathrm{Si}$ & 12 & 25 & \multirow{2}{*}{0.52} & \multirow{2}{*}{0.40} & \multirow{2}{*}{$1.2(0.6-2.7)$} \\
\hline & No & 66 & 175 & & & \\
\hline \multirow{4}{*}{ La jagua de Ibirico } & $\mathrm{Si}$ & 19 & 55 & \multirow[t]{2}{*}{0.59} & \multirow[t]{2}{*}{0.28} & \multirow[t]{2}{*}{$0.85(0.46-1.5)$} \\
\hline & No & 59 & 145 & & & \\
\hline & & & & \multicolumn{3}{|c|}{ Patoco / Patoquilla (Porthidium nasutum) } \\
\hline & & $\mathrm{Si}$ & No & P & $x^{2}$ & OR (IC 95\%) \\
\hline \multirow{2}{*}{ Agustín Codazzi } & $\mathrm{Si}$ & 26 & 73 & \multirow{2}{*}{0.00001} & \multirow{2}{*}{19.08} & \multirow{2}{*}{$4.5(2.2-9.3)$} \\
\hline & No & 13 & 166 & & & \\
\hline \multirow{2}{*}{ Becerril } & $\mathrm{Si}$ & 3 & 33 & \multirow{2}{*}{0.29} & \multirow{2}{*}{1.11} & \multirow{2}{*}{$0.5(0.15-1.8)$} \\
\hline & No & 36 & 206 & & & \\
\hline \multirow{2}{*}{ Chiriguaná } & Si & 3 & 29 & \multirow{2}{*}{0.42} & \multirow{2}{*}{0.64} & \multirow{2}{*}{$0.6(0.17-2)$} \\
\hline & No & 36 & 210 & & & \\
\hline \multirow{4}{*}{ El Paso } & $\mathrm{Si}$ & 0 & 37 & \multirow{2}{*}{0.008} & \multirow{2}{*}{6.96} & \multirow{2}{*}{0} \\
\hline & No & 39 & 202 & & & \\
\hline & & & & \multicolumn{3}{|c|}{ Patoco / Patoquilla (Porthidium nasutum) } \\
\hline & & $\mathrm{Si}$ & No & $\mathbf{P}$ & $x^{2}$ & OR (IC 95\%) \\
\hline \multirow{4}{*}{ La jagua de Ibirico } & $\mathrm{Si}$ & 8 & 66 & 035 & 0.6 & $07\left(\begin{array}{lll}0 & 3 & 1\end{array}\right.$ \\
\hline & No & 31 & 173 & 0.30 & 0.80 & $0.7(0.3-1.5)$ \\
\hline & & & & Cascabe & otalus & \\
\hline & & Si & No & $\mathbf{P}$ & $x^{2}$ & OR (IC 95\%) \\
\hline Aauctín C Cdazi & $\mathrm{Si}$ & 11 & 88 & 00005 & 1106 & $72(10,27)$ \\
\hline Agustini CoudaLt & No & 3 & 176 & 0.0000 & 11.00 & $1.0(1.7-21)$ \\
\hline 드 Doce & $\mathrm{Si}$ & 3 & 34 & 026 & $08 /$ & $18 /(0<0<0)$ \\
\hline Et Paso & No & 11 & 230 & 0.30 & 0.84 & $1.84(0.48-0.4)$ \\
\hline
\end{tabular}

Fuente: elaboración propia.

La georreferenciación de estas ser- de P calculado en el grupo anterior, puepientes en virtud de la frecuencia de los de verse en la figura 1. accidentes ofídicos reportados y el valor 


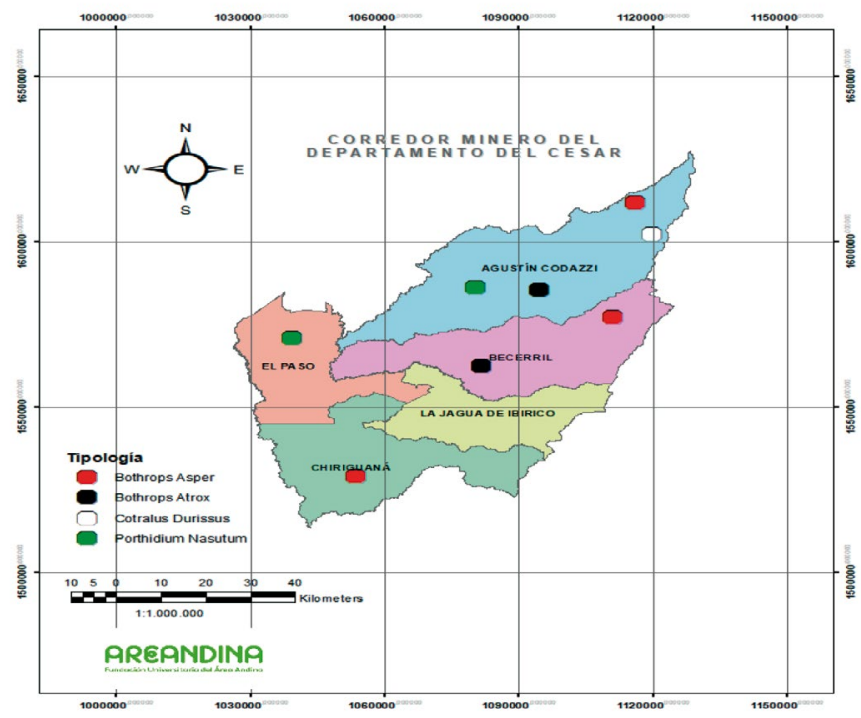

Figura 1. Georreferenciación de las serpientes causantes de accidentes ofídicos en el corredor minero del departamento del Cesar.

Fuente: Secretaría de Salud, (2017 -2019), Departamento del Cesar. Autor: elaboración propia. Nota: Los puntos ubicados en el mapa corresponden a la localización de la fuente de datos disponibles en las fichas de notificación № 100 del INS que sirvieron de base para esa investigación, no se señaló La Jagua de Ibirico debido a no encontrar diferencias significativas para ninguna de las serpientes estudiadas.

Con respecto a la atención inicial, a $36 \%$ de las mordeduras se les aplicó un torniquete; otras medidas incluyeron acciones no médicas como emplastos de hierbas y remedios tradicionales. La tabla 5 muestra el resumen completo de los tipos de atención prehospitalaria recibida.

Tabla 5. Atención inicial recibida.

\begin{tabular}{lccccc}
\hline Acción & 2017 & 2018 & 2019 & Total & $\%$ \\
\hline Torniquete & 35 & 37 & 29 & 101 & $36 \%$ \\
Inmovilización del miembro & 29 & 16 & 22 & 67 & $24 \%$ \\
Otro & 14 & 14 & 10 & 38 & $14 \%$ \\
Inmovilización del paciente & 3 & 5 & 11 & 19 & $7 \%$ \\
\hline
\end{tabular}




\begin{tabular}{lccccc}
\hline Acción & 2017 & 2018 & 2019 & Total & $\%$ \\
\hline Pócimas & 5 & 6 & 6 & 17 & $6 \%$ \\
Emplastos de hierbas & 4 & 4 & 5 & 13 & $5 \%$ \\
Punción & 2 & 4 & 4 & 10 & $4 \%$ \\
Incisión & 2 & 1 & 5 & 8 & $3 \%$ \\
Sangría & 1 & 1 & 3 & 5 & $2 \%$ \\
\hline
\end{tabular}

Fuente: elaboración propia.

La clasificación final de los eventos evidenció que en $49 \%$ de los casos se trató de accidentes leves; $42 \%$ moderados, $5 \%$ severos y no hubo envenenamiento en $4 \%$. Solo hubo dos muertes, equivalente a una letalidad de $0.7 \%$.

\section{Discusión}

Los hallazgos sociodemográficos de esta investigación son similares al reporte de otros autores en diferentes áreas del país. El rango de edades demuestra que los accidentes ofídicos son más comunes desde el inicio de la adultez hasta la quinta década de vida $(7,8,9,10)$, ésta es la edad productiva y la de mayor vinculación laboral de los trabajadores formales e informales; los hombres siguen siendo el género más afectado en una relación 2:1 con respecto a las mujeres $(7,9,11)$, lo cual, posiblemente se deba a que los varones desempeñan con más frecuencia actividades en el campo, son más espontáneos para explorar lugares con vegetación espesa o realizan activi- dades de mayor riesgo que incluyen la manipulación de animales silvestres. No existe diferencia de pertenencia étnica en cuanto a la probabilidad de sufrir un accidente ofídico, como estableció Sevilla-Sánchez et al., en el departamento de Nariño, donde las personas afrodescendientes son el grupo prevalente con $52 \%$ de los casos (9).

Las actividades agrícolas siguen siendo las de mayor riesgo $(8,9,13,14)$. El área de ocurrencia en la mayoría de los casos es rural $(7,8,13)$, no obstante, el número de eventos urbanos va en aumento, posiblemente asociado con desplazamiento de especies por el ingreso del hombre al hábitat de las serpientes, la disponibilidad de un número mayor de presas (animales de corral, ratas y ratones) en asentamientos humanos y fenómenos como el calentamiento global que pueden afectar la alimentación, reproducción y crecimiento de estos animales (15). Actividades como la minería y específicamente la explotación del carbón 
a cielo abierto pueden cambiar algunas condiciones medioambientales como la sensación térmica, depósito de minerales y material de arrastre en fuentes de agua, deforestación de amplias extensiones de bosques, entre otras; todas estas condiciones potenciarían la migración y desaparición de especies alterando los ecosistemas $(16,17)$.

El género de serpientes predominante es el Bothrops, como lo demuestran todos los estudios consultados en el ámbito nacional $(7,8,9,10)$ y en otros países de la región que comparten condiciones climáticas, topográficas y socioculturales $(12,13,14)$. Las especies $B$. asper y B. atrox son predominantes en Colombia, pero su área de influencia se extiende desde América Central hasta Ecuador, por debajo de los $2000 \mathrm{msnm}$ $(12,13,19)$. Los miembros superiores e inferiores son las áreas más afectadas, generalmente, las víctimas no utilizan calzado de corte alto ni emplean guantes de cuero, siendo las manos y los pies susceptibles de mordeduras; esta misma condición, favorece el uso de medidas compresivas como torniquetes pese a que son ampliamente desaconsejados por la comunidad científica $(8,10)$.

Afortunadamente, la mayoría de los eventos se clasificaron como leves o moderados $(7,9,10,12)$ con una letalidad inferior a $1 \%(7,9,14)$, esto último posiblemente debido al uso de antídoto y la oportunidad de recibir atención médica. El avistamiento de los ofidios y el contacto con los humanos lleva a la captura o muerte de los ofidios en un porcentaje variable, que en la literatura se encuentra entre $20-40 \%(9,10,11)$ y en nuestra investigación fue notablemente mayor (54\%); esto último pone en peligro la supervivencia de las diferentes especies y los ecosistemas en los cuales participan.

\section{Conclusiones}

Las mordeduras de serpientes son frecuentes en el área de explotación carbonífera del departamento del Cesar, siendo el género Bothrops spp la más común, principalmente en los municipios de Agustín Codazzi y Becerril en donde se reportaron la mayoría de los eventos. Respecto al tratamiento, la investigación encontró existencia de una cultura ancestral para el uso de remedios naturales o empíricos, no obstante, estas opciones nunca superan la eficacia del suero antiofídico y pueden retrasar la oportunidad de atención médica. Finalmente, muchas serpientes son capturadas o sacrificadas alterando el equilibrio medio ambiental. 


\section{Recomendaciones}

A partir de esta investigación se deriva la imperiosa necesidad de capacitar a propios y extraños sobre identificación, conservación y respeto a la fauna silvestre de la región, incluyendo a las serpientes. Adicionalmente, se debe extremar la prevención primaria, por ejemplo, el uso obligatorio de botas y guantes de cuero mientras se realizan trabajos en áreas rurales. El suero antiofídico es la mejor estrategia para disminuir mortalidad; debería estar disponible en sitios de atención médica de concesiones mineras y hospitales de la región; finalmente, el uso de terapias empíricas como emplastos e infusiones herbales puede ser la base de nuevos proyectos de investigación con el objetivo de refinar principios activos eficaces útiles como terapia coadyuvante del suero antiofídico.

\section{Conflicto de intereses}

Los investigadores manifiestan no tener conflicto de intereses.

\section{Referencias}

Amador, C., Luna, J., \& Puello, E. (2018). Comportamiento de la intoxicación moderada y severa por Ofidiotoxicosis Bothrópica en Córdoba - Colombia. Rev. avances en salud; 2(1): 7-15 Recuperado de: https:// pesquisa.bvsalud.org/portal/resource/pt/ biblio-1087936

Cano, M., \& Popo, J, (2018). Formulación de una metodología para la Restauración Ecológica en ríos afectados por la minería de carbón. Caso de estudio río Lili, Cali. Investigación Formativa en Ingeniería. 2:69-76. Recuperado de: https://www.researchgate. net/profile/Julio_Rivera_Rodriguez/publication/334576100_2018_Implementacion_de_una_estrategia_para_la_minimizacion_de_residuos_quimicos_INGENIO/ links/5d320506299bf1995b397282/2018-Implementacion-de-una-estrategia-para-la-minimizacion-de-residuos-quimicos-INGENIO.pdf\#page $=69$

Congreso de la República de Colombia, (2004). Ley 911. Por la cual se dictan disposiciones en materia de responsabilidad deontológica para el ejercicio de la profesión de Enfermería en Colombia se establece el régimen disciplinario correspondiente y se dictan otras disposiciones. Diario Oficial 45; 693. Recuperado de: https://www.mineducacion.gov.co/1621/articles-105034_archivo_pdf.pdf

Doria, C., \& Fagundo, J. (2017) Niveles de metales en partículas atmosféricas en la zona minera de carbón, Norte de Colombia. 14, (2): 110-121, Recuperado de: http://www. scielo.org.co/scielo.php?script=sci_arttext\&pid=S1692-17982017000200110\&ln$\mathrm{g}=\mathrm{en} \& \mathrm{nrm}=\mathrm{iso}>$. access on 17 May 2020. http://dx.doi.org/10.15332/iteckne. v14i2.1765.

Estrada, S., Quintana, J., \& Varga, L. (2014). Accidente ofídico en animales de pastoreo: acercamiento epidemiológico, clínico y 
de manejo. Rev. Med. Vet., Bogotá, n. 27, p. 149-161. Recuperado de: http://www. scielo.org.co/scielo.php?script=sci_arttext\&pid $=$ S0122-93542014000100013\&ln$\mathrm{g}=\mathrm{en} \& \mathrm{nrm}=\mathrm{iso}>$. access on 16 May 2020 .

Epi Info ${ }^{\text {TM }}$ (2020). La herramienta esencial de software libre para la práctica de la salud pública. Centro para el control y la prevención de enfermedades (CDC). Recuperado de: https://www.cdc.gov/epiinfo/esp/ es_pc.html

Guerra D. (2020). Perfil epidemiológico del accidente ofídico en las tierras bajas de Guatemala. Rev. Ciencia, Tecnología y Salud. 3(2):127. Recuperado de: https://digi. usac.edu.gt/ojsrevistas/index.php/cytes/article/view/112/144

Guzmán, C., Villa, H., Calderón, A. (2020). Análisis epidemiológico y clínico de intoxicaciones agudas atendidas en Montería, Colombia. Revista médica Risaralda 21(1): 17-21. Recuperado de: http://www. scielo.org.co/scielo.php?script $=$ sci_arttext\&pid=S0122-06672015000100005\&ln$\mathrm{g}=\mathrm{en}$.

Instituto Nacional de Salud. (2018). Boletín Epidemiológico Semanal: Semana epidemiológica 46. Recuperado de: https://www.ins.gov.co/buscador-eventos/ BoletinEpidemiologico/2018\%20Bole$\mathrm{t} \% \mathrm{C} 3 \% \mathrm{ADn} \% 20$ epidemiol\%C3\%B3gico\%20semana\%2046.pdf

Leguízamo, T. \& Ruiz, S. (2020). Minería del Carbón: La nueva "joya" de la economía colombiana. Boletín Semillas Ambientales. 12(1):86 Recuperado de: https://revistas.udistrital.edu.co/index.php/bsa/article/ view/13621/13966
Márquez, M., \& Gómez, M. (2015). Ophidic accident in the department of Sucre, Colombia. Nova 13(24):39-46. Recuperado de: http://www.scielo.org.co/pdf/nova/ v13n24/v13n24a04.pdf

Mazzanti, M. (2011) Declaración de Helsinki, principios y valores bioéticos en juego en la investigación médica con seres humanos. Rev Colomb Bioética. 6(1):125-144.

Ministerio de Salud - Colombia, (1993), Resolución 008430. Por la cual se establecen las normas científicas, técnicas y administrativas para la investigación en salud. Recuperado de: https://www.minsalud.gov.co/sites/ rid/Lists/BibliotecaDigital/RIDE/DE/DIJ/ RESOLUCION-8430-DE-1993.PDF

Montoya Domínguez, E. (2018). The extraction of coal in the center of Cesar, Colombia: notes for the understanding of the environmental conflict. Rev. Gestión y Ambiente 21(supl. 2), 62-73. Recuperado de: https://revistas.unal.edu.co/index.php/ gestion/article/view/77836

Oscar, B., Ever, C., \& Douglas, H. (2019). Thermobiology of Bothrops asper (Garman, 1883) in Colombia: ecophysiological tests. Rev Inv Vet Perú. 30(1):61-72. Recuperado de: http://dx.doi.org/10.15381/ rivep.v30i1.15673

Quintero, A., et al. (2015). Desenmascarando el veneno de la serpiente Bothrops asper de Panamá: aislamiento y caracterización de cuatro fosfolipasas A 2. Conference: XIII Congreso Nacional de Ciencia y Tecnología 2010. Recuperado de: http://rgdoi. net/10.13140/RG.2.1.1133.9361 
Sarmiento, K., Torres, I., Guerra, M., Ríos, C., Zapata, C., Suárez, F. (2018). Epidemiological characterization of ophidian accidents in a Colombian tertiary referral hospital. Retrospective study 2004-2014. Rev.fac.med. 66 (2): 153158. Recuperado de: http://www.scielo. org.co/scielo.php?script=sci_arttext\&pi$\mathrm{d}=\mathrm{S} 0120-00112018000200153 \& \operatorname{lng}=$ en . http://dx.doi.org/10.15446/revfacmed. v66n2.61335

Sevilla, M., Mora, D., Calderón, J., Guerrero, J., \& Ayerbe, S. (2019). Accidente ofídico en el departamento de Nariño, Colombia: análisis retrospectivo, 2008-2017. Biomédica. 39(4): 715-736. Recuperado de: http://www.scielo.org. co/scielo.php?script $=$ sci_arttext\&pi$\mathrm{d}=\mathrm{S} 0120-41572019000400715 \& \operatorname{lng}=\mathrm{en}$. http://dx.doi.org/10.7705/biomedica.4830
Vélez, L. (2019). Caracterización del accidente por mordedura de serpiente, atendidos en Unidades de Salud, Zona 5, Ecuador. Revista científica digital INSPILIP. Recuperado de: https://www.inspilip.gob.ec/ wp-content/uploads/2019/02/7-accidentes. pdf

Vera, M., Ríos, C. (2018). Características clínicas y epidemiológicas de los accidentes ofídicos de un hospital regional de Paraguay, 2010 A 2016. Rev. Inst. Med. Trop. 13(2):21. Recuperado de: http://scielo.iics. una.py/scielo.php?script=sci_arttext\&pi$\mathrm{d}=$ S1996-36962018000200021 